\title{
NEPTUNE'S COSMIC RAY CUTOFF
}

\author{
R. S. Selesnick and E. C. Stone
}

California Institute of Technology

Abstract. The Voyager 2 cosmic ray experiment observed the signature of a magnetic cutoff of cosmic ray protons immediately after the closest approach to Neptune. The cutoff signature shows that the simultaneous observation of a trapped particle dropout could not have cocurred at high magnetic latitudes, but is likely to be a dift shadow of the planet caused by the large offset of the tricle drift shells. The OTD2 dipole model of Neptune's metic field predicts a drift shadow at approximately the carrect times. In addition, the cutoff signature is similar to predicted by the OTD2 model, although the model is oc accurate throughout the observation period. The similarity is consistent with small estimated corrections to we predicted signature based on the locally observed magnetic field values, indicating that the $L$ shell values derived from the model are approximately valid after the dosest approach time.

\section{Introduction}

Geomagnetic effects on cosmic rays are well established both observationally and theoretically (see review by Rossi and Olbert [1970] and references therein). A primary effect s the shielding, or cutoff, of cosmic rays from low latitudes with full access at high magnetic latitudes. The close ecounter of Voyager 2 with Neptune provided an opportunity to study this effect in a non-terrestrial planetary magnetic field, and observations by the cosmic ray system (CRS) were first reported by Stone et al. [1989]. They are of particular interest because of the highly complex nature of Neptune's magnetic field [Ness et al., 1989], compared ith the relatively dipolar magnetic field of Earth, providing same insight into the field configuration near the planet.

Both the CRS and low-energy charged particle (LECP) experiments also observed a sharp dropout in the trapped prticle flux near the planet. This feature was interpreted by Krimigis et al. [1989] as evidence for a passage through Neptune's high latitude auroral zone, or polar cap. An explanation, a particle drift shadow due to absorption by Neptune's atmosphere, was suggested by Stone et al. [1989]. As discussed below, the timing of the cosmic ray cutoff provides strong evidence against the polar cap interpretation, and a similar conclusion was reached by Mark et al. [1990] on the basis of the LECP particle igular distributions. In addition, initial quantitative masis of the cutoff feature provides an approximate description of the Voyager 2 location in magnetic $L$ shell minutes following the closest approach to Nepane.

Copyright 1991 by the American Geophysical Union.

Paper number 90GL02582

$0094-8534 / 91 / 90 G L-02582 \$ 03.00$

\section{Data}

Two hours of data from the CRS electron telescope, between 0300 and 0500 SCET (spacecraft event time) on day 237, are shown in Figure 1. The top panel shows the counting rate from a detector (D1) that is responding, with a nearly omnidirectional acceptance, to the flux of electrons with kinetic energies $\geq 1 \mathrm{MeV}$. The middle panel shows the counting rate of a detector (D4) that responds to particles penetrating the significant thickness of passive shielding on each side of the detector. The statistically significant variations in the D4 rate are due primarily to galactic cosmic ray (GCR) protons. There is also a constant background level due primarily to gamma rays from the onboard ${ }^{238} \mathrm{Pu}$ power source, with a smaller contribution from electrons penetrating the sides of the telescope. The GCR protons lose only a small fraction of their energy in penetrating the D4 detector from any direction. The

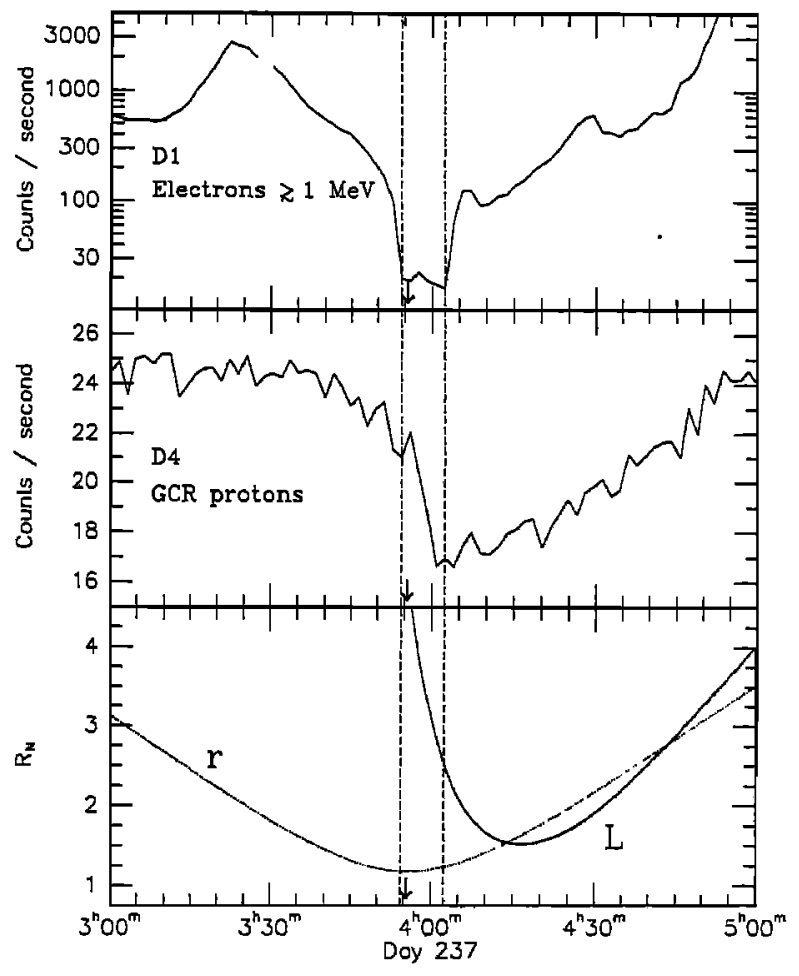

Fig. 1. Counting rates versus time (SCET) from two CRS detectors responding to trapped electrons and galactic cosmic ray (GCR) protons. Also radial distance, $r$, from Neptune and OTD2 $L$ shell of Voyager 2. The vertical dashed lines mark the extent of the flux dropout in the trapped electrons and the arrows mark the time of closest approach to Neptune, 0355:30 SCET at $1.18 \mathrm{R}_{\mathrm{N}}$. The data from the D1 detector are 6 second averages sampled once every 96 seconds. The D4 detector data are 96 second averages sampled continuously. 
detector therefore has only the slight directionality due to its shape, a circular disk with an active area of $4.5 \mathrm{~cm}^{2}$ and a thickness of $3 \mathrm{~mm}$. For a detailed description of the CRS see Stone et al. [1977].

The lower panel of Figure 1 shows the radial distance, $r$, of Voyager 2 from the center of the planet in Neptune radii $\left(R_{N}=24,765 \mathrm{~km}\right)$, and the magnetic $L$ shell parameter derived from the OTD2 dipole magnetic field model (N. F. Ness, private communication). The OTD2 model is similar to the OTD model [Ness et al., 1989] and does not accurately represent the magnetic field data between -0310 and $\sim 0430$ SCET, so that the $L$ values in this period are not necessarily accurate.

\section{Analysis}

A natural explanation for the sharp decrease in the counting rate of the GCR protons near closest approach, followed by the more gradual recovery, is a cosmic ray cutoff. Since the detector is nearly omnidirectional, all arrival directions of cosmic rays are sampled. In the cutoff region certain directions are forbidden to protons arriving from large distances. The solid-angle fraction of forbidden directions at a particular location then determines the relative decrease in the counting rate. A direction may be forbidden either because particles on that trajectory would have intersected the planet before reaching the spacecraft or because the magnetic field allows only a bounded trajectory. The latter effect can be calculated analytically for a dipole magnetic field using the Stormer theory [Stormer, 1955].

At a given magnetic latitude, $\lambda$, distance $r_{d}$ from the dipole center, and for a given cosmic ray arrival direction, represented by the angle $\gamma$ between the velocity vector and the azimuthal vector pointing west, the observable momenta $p$ satisfy the inequality

$$
p>M\left[\frac{1-\left(1-\cos \gamma \cos ^{3} \lambda\right)^{1 / 2}}{r_{d} \cos \gamma \cos \lambda}\right]^{2}
$$

where $M$ is the planetary magnetic dipole moment. For positively charged particles the forbidden directions form a cone about due east.

The predicted cutoff at each point along the Voyager 2 trajectory can be calculated for the OTD2 model using the known spectrum of GCR protons. At high energies the spectrum approximates a power law in energy with spectral index -2.6 , and has been sampled extensively at 1 A.U. [e.g. Simpson, 1983]. The low energy part of the spectrum, which is influenced by spatial gradients in the heliosphere and by solar-cycle modulation, is measured by Voyager 2 . A model of the GCR proton differential flux $(J)$ and integral flux $\left(J_{>E}\right)$ applicable to the Voyager Neptune encounter is shown in Figure 2. The integral flux at low energies determines the counting rate of the D4 detector and is sensitive only to protons with kinetic energies $\geq 100$ $\mathrm{MeV}$. The dashed curves in Figure 2 show the spectra at the minimum OTD2 $L$ reached by Voyager $2(L=1.52$, $\left.r_{d}=1.5\right)$ taking into account the decrease in flux due to the Stormer cone at each energy. The sharp rollover in the differential flux near $10^{4} \mathrm{MeV}$ is centered at the vertical cutoff rigidity. The integral flux at low energies is seen to decrease by a factor of $\mathbf{2 0}$.

The predicted decrease in flux due to the cutoff at any

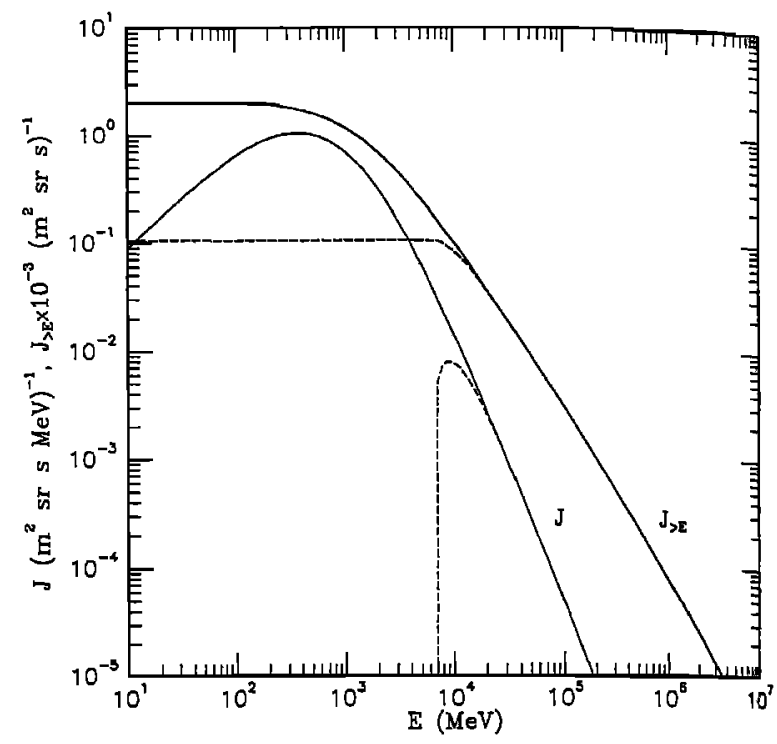

Fig. 2. GCR proton differential flux $(J)$ and integral ftes $\left(J_{>E}\right)$ versus kinetic energy. The dashed curves show the spectra modified by the Stormer cutoff at the position of the Voyager 2 minimum OTD $L$ of 1.52 .

spacecraft location can be combined with the geomeny factor of the D4 detector to compare with the observed decrease in counting rate. Results of the model calculation are shown in Figure 3. The top panel is an approximate calculation of the counting rate decrease due only to shadow effect of the planet. It was made by assuming that the particle trajectories in the vicinity of the planet a straight lines and that any trajectories arriving from with the solid angle subtended by the planet are therefon forbidden. The contribution to the counting rate due secondary cosmic rays (see below) was included in the shadow calculation as they should be reduced by a simin factor to the primaries. The approximation should be adequate for our purposes because, as shown in the figute, the shadow effect is generally small compared to the cuth signature, and it is symmetrical about the closest approct to Neptune whereas the cutoff signature occurs primenity after the closest approach. Although some asymment would be introduced in an accurate calculation of shadow effect, the maximum decrease would be affected.

As shown in the middle panel of Figure 3, the calculated decrease in the counting rate due to the Strimer mapectic cutoff does resemble the observed signanure. The low panel combines the shadow and magnetic cutoffs comparison with the data, shown by the thin line. Tw dashed curves in Figure 3 indicate a modification OTD2 model which is discussed below.

The model results described above show that the content rate decrease is near that expected for a GCR cunfe Estimates of the expected background rate provide independent test of this result. The background doe gamma rays (and any unknown source which is influenced by the GCR flux) is $13 \mathrm{~s}^{-1}$. This number determined by comparing the D4 rate with a mollo coincidence rate that is insensitive to the gamma rays, on the entire Voyager mission, and should be accurat 


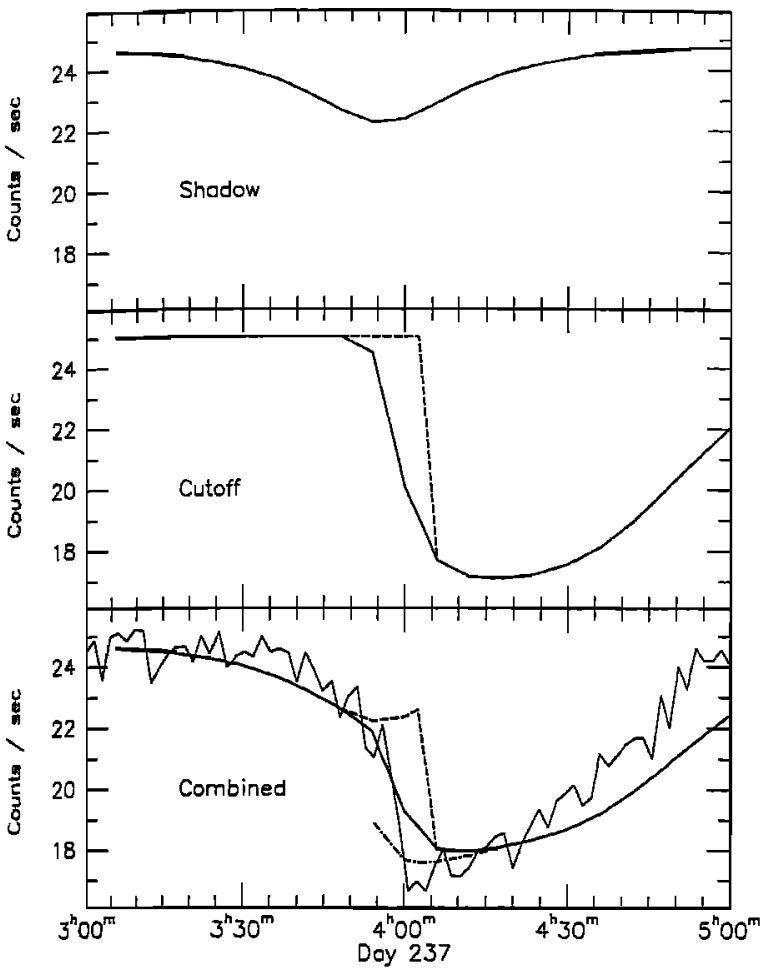

Fig. 3. Model calculations of the D4 counting rate including the effects of planetary shadowing (top), magnetic curoff in the OTD2 model (middle), and total cutoff (combination of shadow and magnetic cutoffs, bottom). The data are shown for comparison by the thin line in the bottom panel. The dashed lines in the middle and lower panels are modifications to the OTD2 predicted cutoff signature for the case of the spacecraft being at high metic latitudes during the trapped flux dropout in Figure 1. The dash-dot line in the lower panel is a correction to the OTD2 model signature based on the locally observed magnetic fields.

within $1 \mathrm{~s}^{-1}$. Another source of background is secondary cosmic rays produced in the spacecraft and telescope. These are potentially the largest source of error in the beckground calculation, but preliminary estimates show that produce $-5 \mathrm{~s}^{-1}$ in $\mathrm{D} 4$ and are primarily due to high mergy primary cosmic rays which are affected by the geometric shadow but not by the magnetic cutoff. With the expected primary cosmic ray rate of $7 \mathrm{~s}^{-1}$, these estimates give a total rate $-25 \mathrm{~s}^{-1}$ outside the cutoff region, consistent with the observations. The flux of GCR helium is $-10 \%$ of proton flux and has been included in the above estimate.

In summary, the analysis described above strongly sapports the hypothesis that the sharp decrease in flux near 0400 is entirely due to a cosmic ray magnetic cutoff. The counting rate decrease is approximately consistent with the substantial loss of GCR proton flux expected for a high rigidity cutoff. This conclusion is independent of backround estimates except for the small expected decrease in the secondary cosmic ray background due to the shadow effect. The background estimates support the conclusion by providing the remainder of the observed counting rate. The thadow effect alone should produce a smaller decrease than observed and depends primarily on the distance from the which changes slowly on the time scale of the flux The spacecraft itself has a negligible shadowing fiect at the GCR energies. Finally, there were no significant changes in spacecraft or detector orientation at the time of the observed decrease. The magnetic latitude, which is the principal factor in determining the cutoff, appears to be the only parameter that could have changed rapidly enough to produce the sharp decrease in flux, and a cutoff appears to be the only effect that could have produced the magnitude of the observed decrease.

\section{Discussion}

The sharp decrease in the CRS D4 rate, corresponding to a rapidly increasing cutoff rigidity, occurred during the dropout of trapped electrons between 0354 and 0402 SCET near the closest approach to Neptune (see Figure 1). In a dipole magnetic field a high rigidity cutoff would be observed only at low magnetic latitudes, so that the flux dropout could then not have been the result of a high magnetic latitude polar cap passage. However, this conclusion applies even to the complex magnetic field of Neptune because the particles near cutoff at high magnetic latitudes are guided by the field lines. The cutoff rigidity of particles observed on field lines which extend into, and have most of their length in, the dipolar field region is determined substantially by the dipole $L$ value in that region [Quenby and Webber, 1959; Sauer and Ray, 1963]. Any difference in the observed cutoff from that predicted by a dipole field model is then due primarily to the separation between the true and model field lines. Therefore the onset of the cutoff signature is an indicator of the time when Voyager was moving from high to low magnetic latitudes, independent of any specific magnetic field model.

The dashed curves in Figure 3 illustrate the minimum modification to the OTD2 cutoff model that would be required if the trapped flux dropout was a signature of a polar cap passage. The observed cutoff signature occurs -5 min earlier than predicted by such a modification.

An altemative to the polar cap explanation of the flux dropout, suggested by Stone et al. [1989], is a drift shadow of the planet, or drift loss cone effect [Roederer, 1970]. In this interpretation, Voyager would have been on particle drift shells which intersected Neptune's atmosphere at some other longitude, causing particles to be absorbed during their drift motion around the planet. The effect is illustrated with the OTD2 model in Figure 4. Contours of magnetic field magnitude (solid curves) and $L$ shell (dashed curves) are shown in a latitude versus longitude projection on the surface of the planet. Also shown is the northern foot of the Voyager 2 field line along its trajectory, with magnetic field magnitudes at the spacecraft indicated near the closest approach. A particle drift shadow is expected at times when the the spacecraft is on drift $(L)$ shells that include surface magnetic field magnitudes smaller than those seen at the spacecraft. From the figure it is evident that the OTD2 model does predict a drift shadow at times nearly coincident with the observed flux dropout (a similar conclusion was reached by Cheng [1990]). For example, during the dropout at -0400 , the local OTD2 magnetic field at the spacecraft when it crossed the $L=3$ shell was $0.069 \mathrm{G}$. The OTD2 surface magnetic field on the same $L$ shell reached a minimum of $0.054 \mathrm{G}$ at $-120^{\circ} \mathrm{W}$. longitude. Since the flux dropout occurred in the region where the OTD2 model is not accurate, more detailed field models will be required to verify the drift shadow interpretation. The coincidence of the end of the dropout at -0402 with the GCR flux 


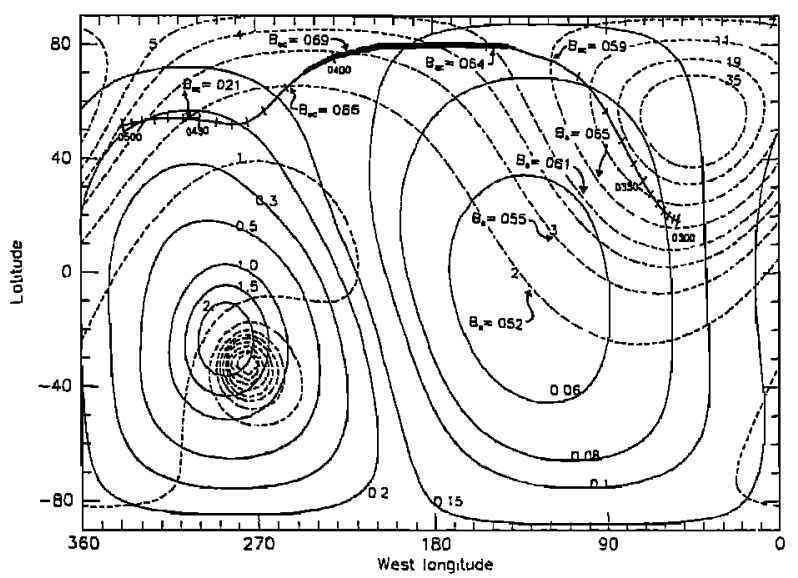

Fig. 4. Contours in latitude versus longitude of the surface magnetic field magnitude (solid curves) and surface $L$ shells (dashed curves) derived from the OTD2 model. The heavy curve is the northern foot of the Voyager 2 field line with tick marks every five minutes from 0300 to 0500 SCET and magnetic field magnitudes at the spacecraft $\left(B_{s c}\right)$ indicated near the dropout. Surface field magnitudes $\left(B_{s}\right)$ are indicated at their minimum values on the same $L$ shells. The extra heavy part of the trajectory is the time of the observed dropout of trapped particle flux and is approximately coincident with the drift shadow that is predicted to be observed whenever $B_{s c}>B_{s}$ on a given $L$ shell. Field magnitudes are in Gauss.

minimum (see Figure 1) does not appear to be of any significance for the model.

Returning to Figure 3, we note that even though the OTD2 model is expected to provide accurate $L$ values only after -0430 SCET, the counting rate based on the OTD2 cutoff shows a similarity to the data throughout the closest approach period. (After -0450 SCET there is probably some response in the D4 detector due to the high trapped electron flux, see Figure 1, so that the comparison may not be valid there.) The reason for the similarity can be understood from the deviations between the observed and model magnetic fields. Quenby and Webber [1959] have derived first order corrections to the dipole cutoff rigidities that depend only on the local field values. These involve a correction to the effective magnetic latitude due to the distortion of the dipole field lines near the planet and, at low magnetic latitudes $\left(<20^{\circ}\right)$, a new effective planetary magnetic dipole moment.

A model cutoff signature, modified from the OTD2 version by the Quenby and Webber corrections using the local magnetic field measurements [N. F. Ness, private communication], is shown by the dash-dot line in Figure 3. The corrections are large $(\Delta L / L>0.5)$ prior to 0355 SCET and are likely uncertain. After 0400 SCET the corrections are smaller $(\Delta L / L<0.4)$ and should be reasonable estimates.

It is encouraging that from -0400 to -0415 the corrected cutoffs are higher than predicted by the OTD2 model, consistent with the observed lower flux levels. The deviations between the observed and model cutoff signatarean from -0430 to -0450 are not presently understoes However, their overall similarity, consistent with the corrections to the model based on the observed magnete field, suggest that the OTD2 $L$ is approximately throughout the period following closest approach. The rait decrease in magnetic latitude expected from the signature prior to -0400 remains to be verified by accarna field models, along with quantitative calculations of cutoff signature for non-dipolar fields by numesical integration of particle trajectories.

Acknowledgements. We thank N. F. Ness for supplyis magnetic field data and the OTD2 model prior publication. This work was supported by NASA contract NAS7-918 and grant NGR 05-002-160.

\section{References}

Cheng, A. F., Global magnetic anomaly and aurora Neptune, Geophys. Res. Let., 17, 1697-1700, 1990.

Krimigis, S. M., et al., Hot plasma and energetic particles Neptune's magnetosphere, Science, 246, 1483-148, 1989.

Mauk, B. H., et al., Energetic charged particle distributiox near $\left(r \leq 2 R_{N}\right)$ and over the pole of Neptune, Geophes Res. Let., 17, 1701-1704, 1990.

Ness, N. F., et al., Magnetic fields at Neptune, Science, 2\% 1473-1478, 1989.

Quenby, J. J. and W. R. Webber, Cosmic ray rigidities and the Earth's magnetic field, Phil. Mas- 4 90-113, 1959.

Roederer, J. G., Dynamics of Geomagnetically Trapd Radiation, Springer, New York, 1970.

Rossi, B. and S. Olbert, Introduction to the Physics of Space, McGraw-Hill, New York, 1970.

Saver, H. H. and E. C. Ray, On Cosmic Ray Cuan Annals Phys., 25, 135-142, 1963.

Simpson, J. A., Elemental and isotopic composition of galactic cosmic rays, in Ann. Rev. Nucl, and Particle Sd. edited by J. D. Jackson, et al. , p. 706, 1983.

Stone, E. C., et al., Cosmic ray investigation for Voyager missions: Energetic particle studies in the heliosphere - and beyond, Space Sci. Rev., 21, 355, 1977,

Stone, E. C., et al., Energetic charged particles in magnetosphere of Neptune, Science, 246, 1489-14t 1989.

Stbrmer, C., The Polar Aurora, Oxford University Pres Fair Lawn, NJ, 1955.

R. S. Selesnick and E. C. Stone, Califomia Instiate Technology, Pasadena, California 91125.

(Received: August 14, 1990;

revised: October 18, 1990;

accepted: November 26, 1990.) 